\title{
Updates in molecular imaging techniques
}

\author{
Hoon Hyun ${ }^{1} \cdot$ Chong-Su Cho ${ }^{2}$
}

Published online: 1 October 2019

(C) The Korean Tissue Engineering and Regenerative Medicine Society 2019

The field of tissue engineering and regenerative medicine (TERM) has made a great progress over the last few decades along with the development of molecular imaging techniques. Non-invasive in vivo imaging techniques including optical imaging, positron emission tomography (PET), magnetic resonance imaging (MRI), and computed tomography (CT) have been used to monitor tissue growth for obtaining longitudinal and quantitative tissue regeneration information in the same animal. Therefore, a strategy for the right combination of molecular imaging techniques and targeted contrast agents has been further applied for diagnosis and therapy of various diseases [1-3].

This special issue contains four review articles that provide recent advances of molecular imaging techniques in the field of TERM. Yang et al. discussed on the use of near-infrared (NIR) imaging system and targeted contrast agents for regenerative medicine. They summarized that the development of NIR contrast agents and their biomedical applications for labeling cells and scaffolds, as well as monitoring the treatment progress of native tissue in living organisms. Importantly, they emphasized that NIR fluorescence imaging techniques combined with targeted contrast agents played a significant role in regenerative medicine by monitoring the therapeutic efficacy of implanted cells and scaffolds which enhanced the

Hoon Hyun

hhyun@jnu.ac.kr

1 Department of Biomedical Sciences, Chonnam National University Medical School, 160 Baekseo-ro, Dong-gu, Gwangju 61469, Republic of Korea

2 Research Institute of Agriculture and Life Sciences, Seoul National University, 1 Gwanak-ro, Gwanak-gu, Seoul 08826, Republic of Korea development of cell therapies and promoted their successful clinical translations [4]. Jung et al. summarized the current state of knowledge in bone tissue-specific NIR imaging and preclinical application for diagnostic purpose. They discussed on the use of bone-targeting NIR contrast agents and a novel strategy of structure-inherent chemical recognition in bone-targeted imaging potential for clinical use. They suggest that rational design of NIR contrast agents by considering molecular properties, target specificity, biodistribution, and targeting mechanism is the only way to overcome the limitations for clinical use of NIR contrast agents [5]. Pillarisetti et al. provided an overview of the recent advances in composite iron oxide nanoparticles (CIONPs) for multimodal imaging and focused on the therapeutic applications of CIONPs. They highlighted that several examples related to the formation, biofunctionalization, and modification of CIONPs for sensitive T1/T2weighted MRI, MR multimodal imaging studies, and therapeutic approaches. They concluded that multifunctional CIONP-based applications could be extended for theranostics of diverse medical diseases in the future after studying the biosafety issues [6]. Kim et al. summarized recent advances of biomaterial-based approaches for the treatment of injured tendons. They concluded that future studies should make more efforts to find the optimum combinations of regenerative factors and ideal biomaterials, providing huge number of possibilities for their clinical applications. Although more comprehensive evaluations of the structural, compositional, and functional changes between molecular imaging tools and histological data are still required, the application of these imaging tools will help to better understand the structural and functional characteristics of scaffolds or tissues for many clinical applications [7]. 
The goal of this special issue is to provide an overview of the emerging molecular imaging techniques and trends in TERM focused on the most promising healthcare technologies for the future of patients care. We believe that molecular imaging techniques are playing an important and critical role in supporting various theranostic applications in clinic.

Acknowledgements The editors express a deep sense of gratitude to all the invited authors for their valuable efforts for the review articles.

\section{References}

1. Kupfer ME, Ogle BM. Advanced imaging approaches for regenerative medicine: emerging technologies for monitoring stem cell fate in vitro and in vivo. Biotechnol J. 2015;10:1515-28.

2. Choi HS, Frangioni JV. Nanoparticles for biomedical imaging: fundamentals of clinical translation. Mol Imaging. 2010;9:291-310.
3. Yahyapour R, Farhood B, Graily G, Rezaeyan A, Rezapoor S, Abdollahi $\mathrm{H}$, et al. Stem cell tracing through MR molecular imaging. Tissue Eng Regen Med. 2018;15:249-61.

4. Yang C, Park GK, McDonald EJ, Choi HS. Targeted near-infrared fluorescence imaging for regenerative medicine. Tissue Eng Regen Med. 2019. https://doi.org/10.1007/s13770-019-00219-6.

5. Jung JS, Jo D, Jo G, Hyun H. Near-infrared contrast agents for bone-targeted imaging. Tissue Eng Regen Med. 2019. https://doi. org/10.1007/s13770-019-00208-9.

6. Pillarisetti S, Uthaman S, Huh KM, Koh YS, Lee S, Park IK. Multimodal composite iron oxide nanoparticles for biomedical applications. Tissue Eng Regen Med. 2019. https://doi.org/10. 1007/s13770-019-00218-7.

7. Kim SE, Kim JG, Park K. Biomaterials for the treatment of tendon injury. Tissue Eng Regen Med. 2019. https://doi.org/10.1007/ s13770-019-00217-8.

Publisher's Note Springer Nature remains neutral with regard to jurisdictional claims in published maps and institutional affiliations. 